\title{
Quality of Service based Performance Metrics on Routing Strategies in MANET with Few Nodes
}

\author{
Jagadish Gurrala \\ Dept. of CSE \\ Anil Neerukonda Institute of \\ Technology and Sciences \\ Sangivalasa, Visakhapatnam
}

\author{
B Ravi Kiran \\ Dept. of CSE \\ Anil Neerukonda Institute of \\ Technology and Sciences \\ Sangivalasa, Visakhapatnam
}

\author{
Mandava Kranthi Kiran \\ Dept. of CSE \\ Anil Neerukonda Institute of \\ Technology and Sciences \\ Sangivalasa, Visakhapatnam
}

\begin{abstract}
Routing strategy in MANET is a tedious job due to changing its backbone over time. Since mobile ad hoc network is a set of independent nodes move freely through radio signals, from which all of them are created for the purpose of data transfer. We need efficient routing protocol is needed to guide signals from source to destination through multiple hops with less delay and several routing strategies were proposed in ad-hoc network for routing of packets through many links. In this paper, we obtain results of performance comparison of four routing strategies used to determine efficiency from thoroughly running simulations on analyzer and produce relative values of specified proactive type routing protocols and specified reactive type routing protocols with few nodes. Finally we were check out performance evaluation of the routing protocols on limited mobile nodes have been serviced to achieve Quality of routing using Qual-Net Simulator and summarize the results.
\end{abstract}

\section{General Terms}

Routing Strategy, Packet, Signal, Node, MANET, QualNet

\section{Keywords}

Throughput, Average End to End delay, Average Jitter, FSR,DSR,DYMO,STAR-LORA.

\section{INTRODUCTION}

In the mid of twenty first century, several researchers have conducted comparative study and performance evaluation [6][7][8] among several routing protocols(sometimes called strategy) with respect to performance metrics over nodes ranges from 50 to 200 were deployed in MANET. In recent years, there have been a lot of articles are published regarding routing protocols in the field of wireless mobile ad hoc networks using ns2 simulator and QualNet simulator. But there is lack of usage of MANET in real time situations. In this paper we leave efficient routing strategy to public from obtaining results through comparative study between proactive type Fisheye State Routing Protocol (FSR), proactive type Source Tree Adaptive Routing protocol(STAR) and Reactive type Dynamic Source Routing(DSR) and Reactive type Dynamic MANET On demand Routing Protocol(DYMO) are analyzed and presented and left to implementers whatever ease of routing technology is available in which how packets are delivered without Access Point. With this paper to explores the impact of QoS metrics such as throughput, average end to end delay and average jitter on routing between nodes where we applied under Constant Bit Rate(CBR) client server traffic conditions using QualNet
5.0.2 simulator[13]. To show the performance efficiency in terms of plotting graphs represents that $\mathrm{X}$ axis indicates Node Count starting from 2 nodes to 12 nodes and $\mathrm{Y}$ axis indicates the Quality of Service metric such as throughput, average end to end delay and average jitter.

The key factor that determines, how efficiently a multi-hop wireless network reacts to topology(backbone) changes frequently based on bandwidth on demand. Routing is performed for many kinds of networks, including the public switched telephone network (PSTN), electronic data networks (such as the Internet) and transportation networks[8]. To deal with certain performance metrics nodes need to know demand on routes which laid a path to receiver in the network.

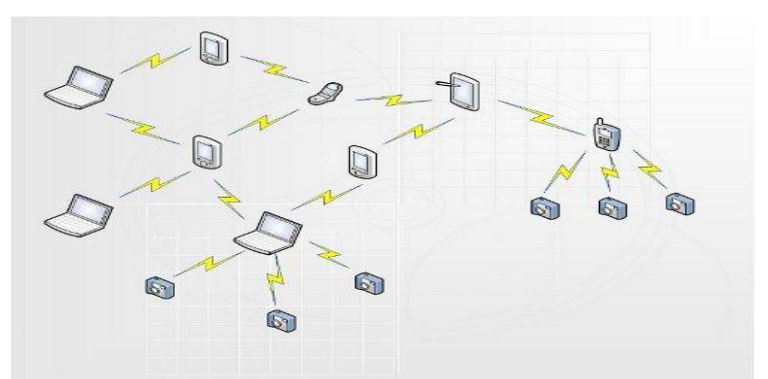

Figure 1 Mobile Ad hoc Network environment

The rest of this paper is organized as follows. In section 2 brief introduction and its literature review on various routing strategies were presented. In section 3 focuses on related work and its test cases on routing. Simulation environment, simulation setup and running a scenarios were discussed in section 4. In section 5 the results of the performance evaluation are discussed. Conclusion \& Future work is given in section 6 .

\section{ROUTING STRATEGY}

Routing protocol in MANET is the prototype in which determination of selecting path by initiation of each node(especially source node) to route packets to concerned destination over wireless media based on CBR traffic. For this work focuses on efficiency of routing protocol among protocols for better throughput and minimize delay in mobile ad-hoc networks. Each node[7] participates in an ad hoc routing protocol that allows it to discover "multi-hop" paths through the network to any other node. Some researchers proposed another article about routing is concerned with in electronic data networks which uses packet switching technology[8]. 
The fundamental idea of a routing protocol is to deliver the messages from source to destination with enhanced performance in terms of minimization of delay. Routing protocols are generally necessary for maintaining effective communication between distinct nodes. Routing protocol not only discovers network topology but also built the route for forwarding data packets and dynamically maintains routes between any pair of communicating nodes. Routing protocols are designed to adapt frequent updates in the network due to mobility nature in MANET.

Conventionally, MANet Routing protocol strategies are implemented by two ways as shown in fig 2 .

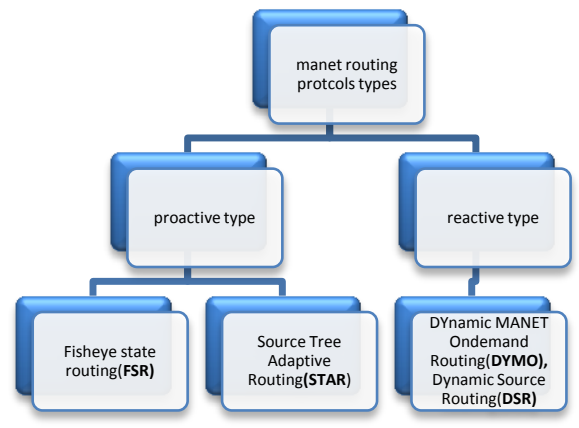

Figure 2 TYPES of MANET routing protocols

\subsection{PROACTIVE ROUTING STRATEGY}

Proactive Routing Strategy also called table driven, or data gram approach in packet switching network. It maintains routing table using the routing information learnt from neighbours on periodic basis. Main characteristics of these protocols include: distributed, shortest-path protocols, maintain routes between every host pair at all times, based on periodic updates of routing table and high routing overhead and consumes more bandwidth[9]. We are discuss comparative analysis between the following strategies in detail. Now we discuss the following kinds in this strategy.

1. Source Tree Adaptive Routing (STAR) []

2. Fisheye State Routing (FSR) [2]

\subsubsection{Fisheye State Routing (FSR)}

The FSR protocol[2] is the next generation technology of Global State Routing strategy(GSR). FSR maintains Entries of nearby nodes in the routing table are updated and exchanged with neighbours more frequently (to reduce the update message size).The accuracy of route increases as packets gets closer to the destination. The main drawback of FSR is as the mobility of remote nodes increases the accuracy of the routing information decreases.

\subsubsection{Source -Tree Adaptive Routing (STAR)}

The STAR protocol [10][11] is also called STAR LORA(Least Overhead Routing Adaptive) based on the link state algorithm. Each router maintains a source tree, which is a set of links form a graph $(\mathrm{V}, \mathrm{E})$ containing the preferred paths to destinations. This protocol has significantly reduced the amount of routing overhead disseminated into the network by using a least overhead routing approach (LORA), to exchange routing information. It also support optimum routing approach (ORA) if required. This approach eliminated the periodic updating procedure present in the Link State algorithm by making update dissemination conditional. Each node keeps the state of subset of the links, i.e., the links of the source trees of other nodes and all of its links. The source tree of a node is the tree composed of all links that constitute the preferred routes to all destinations. STAR based on the trees reported by neighbours and the state of its links, each node builds a partial topology graph that is used to build the routing table. STAR is a scalable algorithm.

\subsection{REACTIVE ROUTING STRATEGY}

Reactive Routing strategy [4] are also called demand driven approach or virtual circuit approach in packet switching network. that find path as and when required. They maintain information about the active routes only. They performs route discovery phase before data transmission by flooding route request packet and destination node reply with route reply packet. A separate route maintenance procedure is required in case of route failure. Main Characteristics of these routing protocols are: determine routes as and when required, less routing overhead, source initiated route discovery and more route discovery delay. The following are used for this kind.

1. Dynamic MANET On-Demand Routing (DYMO) [3]

2. Dynamic Source Routing (DSR) [10]

\subsubsection{DYnamic MANET On-Demand Routing (DYMO)}

Dynamic MANET On-demand (DYMO)[3] routing protocol is a source initiated or reactive routing strategy in which multi hop routing is built up between participating nodes that wish to communicate. The basic operations of the DYMO protocol are route discovery and route maintenance. During route discovery the originating node initiates dissemination of a Route Request (RREQ) throughout the network to find the target node. During this dissemination process, each intermediate node records a route to the originating node. When the target node receives the RREQ, it responds with a Route Reply (RREP) unicast toward the originating node. Each node that receives the RREP records a route to the target node, and then the RREP is unicast toward the originating node. When the originating node receives the RREP, routes have then been established between the originating node and the target node in both directions. During route maintenance, all nodes maintain their routes and monitor their links. When a packet is received for a route that is no longer available the source of the packet is notified. A Route Error (RERR) is sent to the packet source to indicate the current route is broken. Once the source receives the RERR, it re-initiates route discovery if it still has packets to deliver.

\subsubsection{Dynamic Source Routing (DSR)}

DSR is an on demand routing protocol in which a sender determines the exact sequence of nodes through which a packet is propagated. The packet header contains a list of intermediate nodes for routing. Route cache is maintained by each node which caches the source route that it has learned.

The major components of DSR are "Route Discovery" and "Route Maintenance" which work together for determining and maintaining routes to arbitrary destinations [1]. It is designed to restrict the bandwidth consumed by control packets in ad hoc wireless networks by eliminating the periodic table-update messages required in the table-driven approach. A route is established by flooding Route Request packets in the network. [5]. 


\section{RELATED WORK}

In this paper, we have taken two different scenarios. In the first scenario, traffic pattern is taken as CBR Client and no. of nodes have been varied and performance comparisons have been made between STAR-LORA,FSR and DYMO,DSR protocol. In the second scenario, traffic pattern is taken as CBR Server have been varied and performance comparisons have been made between STAR-LORA,FSR and DYMO,DSR protocols.. The QoS can be defined as the manner that the service of delivery of packages is supplied and who can be characterized by various parameters of performance like, the throughput, the delay variation (jitter).

\subsection{Test Scenario 1}

In first scenario we have taken CBR Client as traffic pattern. Parameters are specified in table 1

Table 1: Parameters for Scenario 1

\begin{tabular}{|c|c|}
\hline Parameter & value \\
\hline Terrain size & 1500 X 1500 \\
\hline Number of nodes & $2,4,6,8,12$ \\
\hline Traffic type & Constant Bit Rate client \\
\hline Packet size & 512 \\
\hline Mobility & Random way \\
\hline Speed & 100 mps \\
\hline Pause time & 15,20,25 \\
\hline Simulation time & 3000 sec \\
\hline Routing protocol & FSR,STAR- \\
& LORA,DYMO,DSR \\
\hline
\end{tabular}

\subsection{Test Scenario 2}

In first scenario we have taken CBR Server as traffic pattern. Parameters are specified in table 2

Table 2: Parameters for Scenario 2

\begin{tabular}{|c|c|}
\hline Parameter & value \\
\hline Terrain size & 500 X 500 \\
\hline Number of nodes & $2,4,6,8,12$ \\
\hline Traffic type & Constant Bit Rate Server \\
\hline Packet size & 512 \\
\hline Mobility & Random way \\
\hline Speed & 100 mps \\
\hline Pause time & 15,20,25 \\
\hline Simulation time & 300sec, 3000 sec \\
\hline Routing protocol & FSR,STAR- \\
& LORA,DYMO,DSR \\
\hline
\end{tabular}

\section{SIMULATION ENVIRONMENT}

The QualNet 5.0.2 simulator is used for the design of scenarios in graphical environment. To[15] give an idea of how (in terms of quantitative) the scenario performs it can be run using the QualNet Animator. Figure 3 shows the Animator with the previously designed scenario in action. On the right side you can enable or disable various types of animations. In the Layers tab animations for each of the seven OSI layers can be enabled or disabled individually.

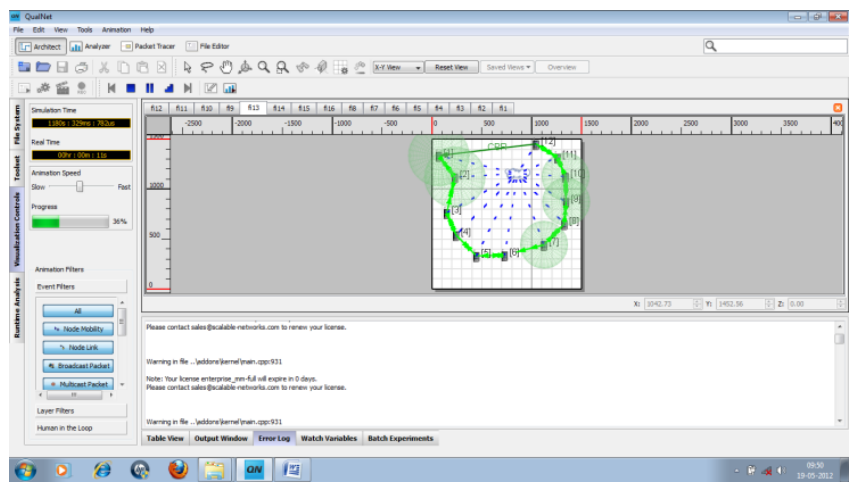

Figure 3 Snap shot of Qualnet Animator in Action

\subsection{EXPERIMENTAL SETUP}

The study has been done to place require number of nodes changes dynamically on canvass plane as shown in Fig 3 and compare the efficiency of four different routing protocols, two of them are from proactive type and rest of them are reactive type in Mobile Ad hoc Networks. The Quality of Service parameters are Throughput, Average End to End Delay and Average Jitter. The above simulations are tested with respect to $2,4,6,8$ and 12 nodes. The performance of all four routing protocols is carried out and results are compiled.

\subsection{PERFORMANCE METRICS}

Now we are conducted extensive calculation on metrics based on terrain size. Hence terrain size varies, the corresponding metrics are rapidly changes while number of nodes are fixed. Here we perform rigorous experimental scenarios are simulated in QualNet simulator to generate graphs in terms of metrics. The following metrics are studied and applied to current scenarios as shown in table 3, 4 and 5 .

\subsubsection{Average end to end delay}

It is the average time it takes a signal travel from process at source node service access point(SAP) to process at destination node service access point(done at SAP of transportation layer in TCP/IP protocol suite). This metric is calculated by subtracting time at which first packet was transmitted by source from time at which first data packet arrived to destination. This metric is significant in understanding the delay introduced by path discovery.

\subsubsection{Throughput}

The throughput of the protocols can be defined as total number of signalling elements travelled at a unit time. It is the amount of data per time unit that is delivered from one node to another via a communication link. The throughput is measured in bits per second (bit/s or bps). 


\subsubsection{Average Jitter}

Jitter is the variation in delay for packets belonging to same flow. In average jitter calculation the variation in the packet arrival time is expected to be low. The delays between the different packets need to be low for better performance in adhoc networks. It becomes a matter of concern if it is more than the threshold value, which is different for data, voice or video transmission services.

\subsection{RESULTS \& COMPARISONS}

Table. 3 CBR client

Througput (Bits/sec)

\begin{tabular}{|c|c|c|c|c|}
\hline nodes \# & FSR & $\begin{array}{c}\text { STAR- } \\
\text { LORA }\end{array}$ & DYMO & DSR \\
\hline 2 & 4274 & 4274 & 4274 & 8520 \\
\hline 4 & 4274 & 4274 & 4274 & 8520 \\
\hline 6 & 4274 & 4274 & 4274 & 8520 \\
\hline 8 & 4274 & 4274 & 4274 & 8520 \\
\hline 12 & 4274 & 4274 & 4274 & 8520 \\
\hline
\end{tabular}

Table. 4 CBR Server Average End to End delay (s)

\begin{tabular}{|c|c|c|c|c|}
\hline $\begin{array}{c}\text { Nodes } \\
\#\end{array}$ & FSR & $\begin{array}{c}\text { STAR- } \\
\text { LORA }\end{array}$ & DYMO & DSR \\
\hline 2 & 0.003721 & 0.004988 & 0.001978 & 0.026 \\
\hline 4 & 0.003734 & 0.004988 & 0.018556 & 0.026 \\
\hline 6 & 0.003756 & 0.004988 & 0.01964 & 0.139 \\
\hline 8 & 0.003769 & 0.00698 & 0.018 & 0.139 \\
\hline 12 & 0.003794 & 0.004789 & 0.02164 & 0.04587 \\
\hline
\end{tabular}

Table. 5 CBR Server Average Jitter (s)

\begin{tabular}{|c|c|c|c|c|}
\hline $\begin{array}{c}\text { Node } \\
\text { S \# }\end{array}$ & FSR & $\begin{array}{c}\text { STAR- } \\
\text { LORA }\end{array}$ & DYMO & DSR \\
\hline 2 & $\begin{array}{c}0.00032 \\
88\end{array}$ & $\begin{array}{c}0.000158 \\
67\end{array}$ & $\begin{array}{c}0.01286 \\
54\end{array}$ & $\begin{array}{c}0.01356 \\
89\end{array}$ \\
\hline 4 & $\begin{array}{c}0.00032 \\
87\end{array}$ & $\begin{array}{c}0.000158 \\
69\end{array}$ & $\begin{array}{c}0.01262 \\
61\end{array}$ & $\begin{array}{c}0.03214 \\
5\end{array}$ \\
\hline 6 & $\begin{array}{c}0.00033 \\
24\end{array}$ & $\begin{array}{c}0.000158 \\
74\end{array}$ & $\begin{array}{c}0.01296 \\
4\end{array}$ & $\begin{array}{c}0.01589 \\
4\end{array}$ \\
\hline 8 & $\begin{array}{c}0.00033 \\
65\end{array}$ & $\begin{array}{c}0.000158 \\
76\end{array}$ & 0.013 & $\begin{array}{c}\text { (6) } \\
12\end{array}$ \\
\hline $\begin{array}{c}0.00033 \\
81\end{array}$ & $\begin{array}{c}0.001058 \\
65\end{array}$ & & 0.01625 \\
7
\end{tabular}

Note: The above calculated result derived from graph analyser screen in Qualnet and it is plotted with the help of
Microsoft Excel 2007 tool. Thus to plot these values in Excel. Figures 5,6, 7,8,9 and 10 shown figures $0,5,10,15$ in $\mathrm{X}$ axis instead of display figures $2,4,6,8,10$ and 12 nodes on $X$ axis in Qualnet experimentation. Readers can understood these numbering notations.

\subsection{RUNNING A SCENARIO}

QualNet 5.0.2 has a configuration window which contains several attributes of each node layer information. However we have to focus on network layer protocols such as DYMO, STAR,DSR and application layer protocol such as FSR. To run each protocol, first of all we are loading values of simulation time, number of seeds (here only one seed is used in simulator) and throughput, average end to end delay, average jitter, then apply run simulation and play it then automatically the present scenario is get animated. Results are obtained from the following analyzer window.

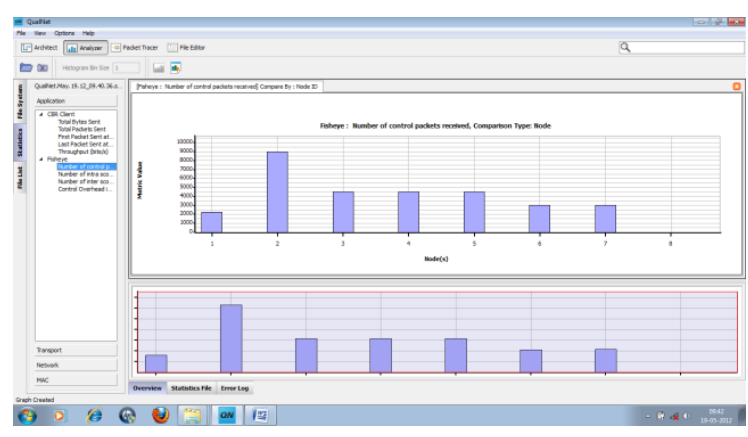

Figure 4 Metric type Vs node ID in Graph Analyzer

\section{PERFORMANCE EVALUATION}

The Qualnet 5.0.2 network simulator[14] has been used to analyze the parametric performance of Source Tree Adaptive Routing Protocol (STAR), Fisheye State Routing Protocol (FSR) and Dynamic Source Routing (DSR),Dynamic MANET On demand Routing protocol (DYMO). The metric based evaluation is shown in figure 5 to figure 10 .

\subsection{Throughput}

With the varying CBR data traffic the throughput is analyzed. The successful packet delivery at given nodes having node identification(ID) in an ad hoc network is observed with increasing MAC based traffic load and mobility. It is found that DSR with least routing overhead uniformly performs better than FSR and STAR The performance related graphs are shown in figure 5 and 6.

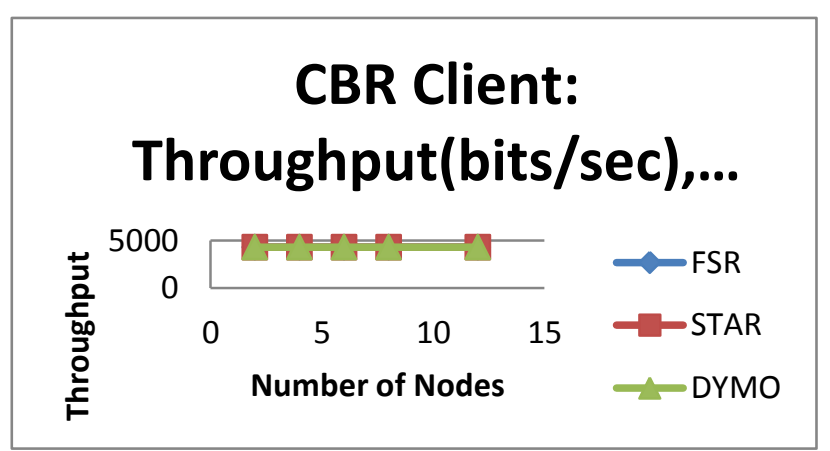

Fig. 5 Graph for Throughput(Bits/sec) Vs nodes 


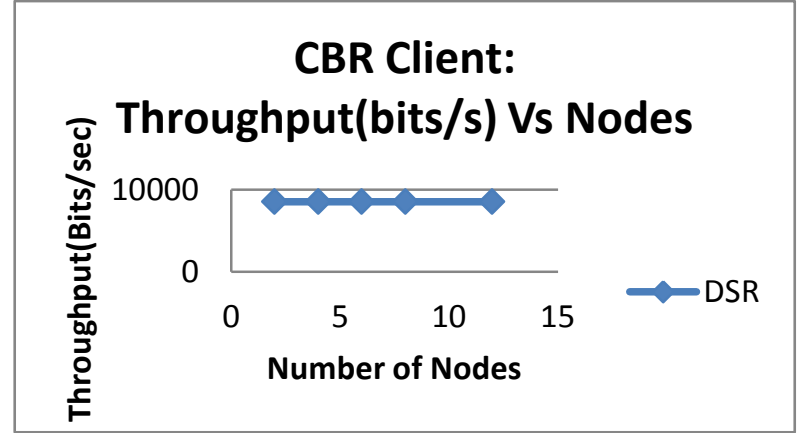

Fig. 6 Average Throughput(Bits/s) Vs nodes

\subsection{Average End-to-End Delay}

it is a propagation time to deliver packet or signal from process (Application Programming Interface) at source to process at destination. To average up all times when data transfer phase is completed that time is called average end-toend delay. Simply we call setup time+data transfer time+tear down time is referred to as delay of packet delivery. In this analysis it is observed as expected the delays are high in DSR in comparison to FSR and STAR. These delays are incurred by the FSR methods. The end-to-end delay is very less in case of FSR and STAR. Because FSR is a less over head to the packet within small geographical area. The performance is shown in figure 7 and 8

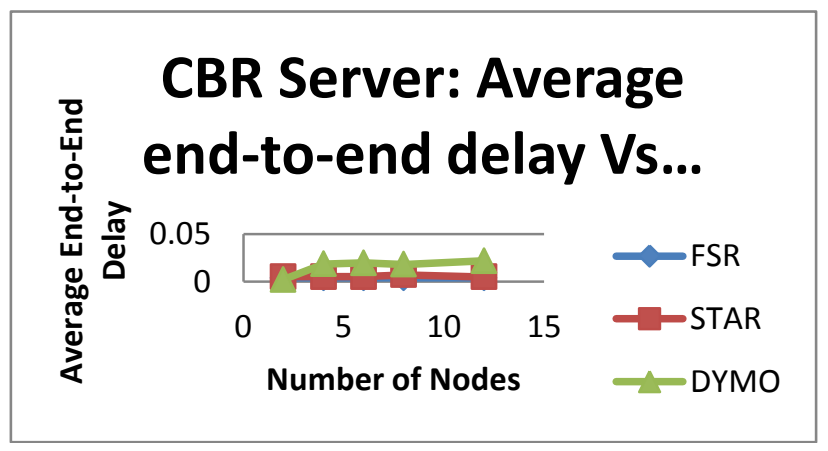

Fig. 7 Average End-to-End delay Vs nodes

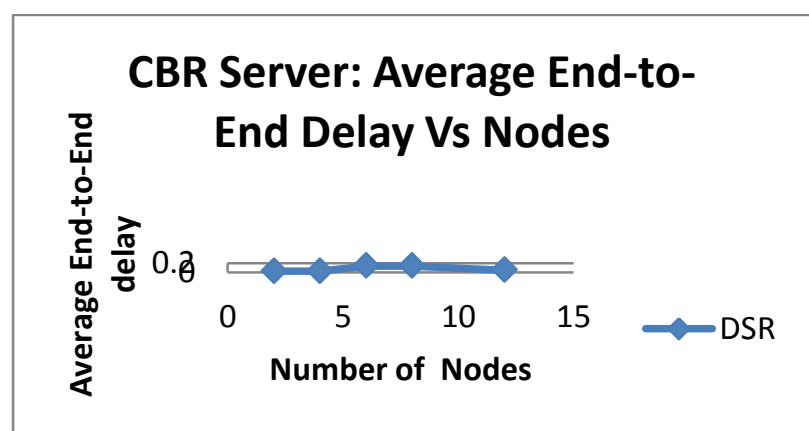

Fig. 8 Average End-to-End delay Vs nodes

\subsection{Average Jitter}

Jitter, the variation of the packet arrival time, is an important metrics for any routing protocol. In this analysis it is found to vary. Initially it is low but for higher nodes ID than 12 it is high. The jitter for nodes 2,4,6,8 is high for both of the protocols due to larger distance between source and destination. In STAR it is due to limited no.of nodes as in FSR and DYMO, it is due to higher frequency of propagation. The jitter results are shown in figure 9 and 10

Fig. 9 Average Jitter Vs nodes

\section{CBR Server: Average Jitter Vs Nodes}

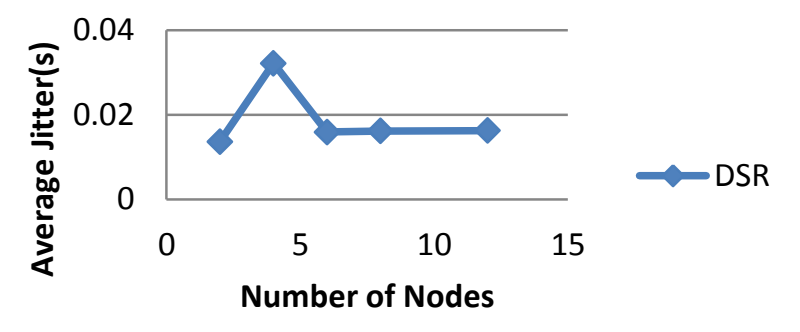

Fig. 10 Average Jitter (s) Vs nodes

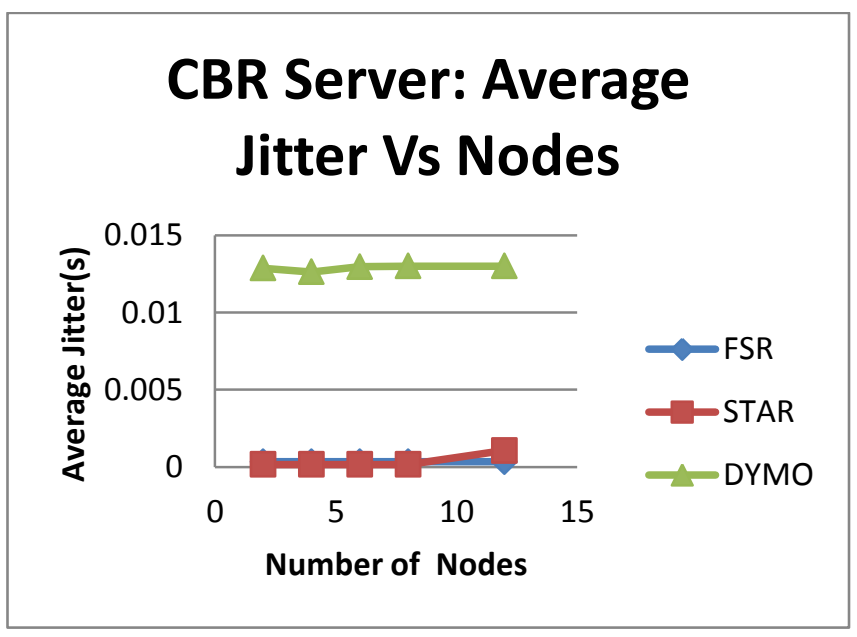

\section{CONCLUSION \& FUTURE WORK}

The performance evaluation of two proactive routing strategies(FSR,STAR) and two reactive routing strategies (DSR, DYMO) for stationary nodes are evaluated by varying the node density $(2,4,6,8,10,12)$ using QualNet 5.0.2network simulator. From the graphs it can be realised that reactive routing protocol DSR only are suited for applications where average jitter and throughput are in danger situation. The worst drawback [15] of the QualNet animator is its extreme high CPU utilization and its implementation in Java which makes it run very slowly on most machines. In this paper leaves to researchers to enhance our mission to run scenarios not only these tools usage. However there is scope of examination over strategies for best effort delivery. One of our future research works is to develop an proposed algorithm on routing strategy can work in Bandwidth on demand environment like banking sector, high secure communication, education system using high end tools. 


\section{REFERENCES}

[1] D. Johnson and D. Maltz. "Dynamic source routing in ad hoc wireless networks", In T. Imielinski and H. Korth, editors,Mobile computing, chapter 5. Kluwer Academic, 1996.

[2] G. Pei, M. Gerla, and T. W. Chen, "Fisheye State Routing in Mobile Ad Hoc Networks," In Proceedings of the 2000 ICDCS workshops, Taipei, Taiwan, Apr. 2000.

[3]http://moment.cs.ucsb.edu/pub/draft-ietf-manet-dymo02.html

[4] Garcia-Luna-Aceves, C. Marcelo Spohn, Source-tree routing in wireless networks, in: Proceedings of the Seventh Annual International Conference on Network Protocols Toronto, Canada, October 1999, p. 273.

[5]Gergely Acs, Levente Buttyan and Istvan Vajda " Provably Secure on-demand Source routing in Mobile Ad-Hoc Networks" Accepted for publication in the IEEE transactions on Mobile Computing, November 28, 2005

[6] M.Uma, G.Padmavathi, "A Comparative Study and Performance Evaluation of Reactive Quality of Service Routing Protocols in Mobile Advhoc networks", Journal of Theoretical and Applied Information Technology, copyright $2005-2009$.

[7] Parma Nand, S.C. Sharma, "Comparative study and Performance Analysis of FSR, ZRP and AODV Routing Protocols for MANET", 2nd International Conference and workshop on Emerging Trends in Technology (ICWET) 2011 Proceedings published by International Journal of Computer Applications ${ }^{\circledR}$ (IJCA).
[8] Subramanya Bhat.M, et al.," A Performance Study of Proactive, Reactive and Hybrid Routing Protocols using Qualnet Simulator", International Journal of Computer Applications (0975 - 8887) Volume 28- No.5, August 2011

[9] Ravinder Ahuja, "Simulation based Performance Evaluation and Comparison of Reactive, Proactive and Hybrid Routing Protocols based on Random Waypoint Mobility Model", International Journal of Computer Applications (0975 - 8887) Volume 7- No.11, October 2010.

[10] Josh Broch, David Johnson, and David Maltz. "The dynamic source routing protocol for mobile ad hoc networks for IPv4 IETF RFC 4728, Feb 2007.

[11] Parma Nand, S.C. Sharma, Rani Astya, "Traffic Load based Performance Analysis of DSR, STAR \& AODV Ad hoc Routing Protocol", International Journal of AdvancedComputer Science and Applications, vol 1 No 4, pp 58-62,Oct 2010.

[12] Suresh Kumar, R K Rathy and Diwakar Pandey, "Traffic pattern based performance comparison of two reactive routing protocols for ad hoc networks using NS@\|, (C) 2009 IEEE.

[13] Qualnet Simulator www.scalable-networks.com

[14] Parma Nand, S.C. Sharma, "Performance study of Broadcast based Mobile Adhoc Routing Protocols AODV, DSR and DYMO", International Journal of Security and Its Applications, vol.5 No. 1, pp 53-64, January, 2011.

[15]ftp://ftp.tik.ee.ethz.ch/pub/publications/TIK-Report 255.pdf,page11 